\title{
177. Intraarterielle Chemotherapie mit intermittierender Hypoxie bei disseminierten Lebermetastasen colorektaler Carcinome
}

\author{
F. Wopfner \\ Chirurgische Universitätsklinik Erlangen (Dir.: Prof. Dr. F. P. Gall), Maximiliansplatz, D-8520 Erlangen
}

\section{Intra-arterial Chemotherapy with Transient Hypoxia in Disseminated Colorectal Liver Metastases}

Summary. In 37 patients suffering from disseminated liver metastases from colorectal primaries, who had undergone a local radical operation and had no other distant metastases, intra-arterial chemotherapy was performed ambulatory during a 30-min block via special catheters. During an average treatment time of 10 months until thrombosis with 5-FU (average of $1000 \mathrm{mg} /$ week), the median survival of all stages (Lausanne) was 14 months, the 1-year survival rate $76 \pm 16 \%$ (act. surv.). Dosage was regulated according to the patients individual tolerance and showed minimal toxicity by careful dose escalation. This kind of treatment provides safe, effective local palliation with increased quality of life.

Key words: Disseminated liver metastases - Intra-arterial chemotherapy - Transient intraluminary hypoxia.

Zusammenfassung. Bei 37 Patienten mit disseminierten Lebermetastasen lokal radikal operierter colorectaler Carcinome ohne sonstige Fernmetastasen wurden eine intraarterielle Chemotherapie während eines dreißigminütigen Blocks mittels eines speziellen Katheters durchgeführt. Bei einer Behandlungszeit von im Mittel 10 Monaten bis zur Thrombosierung mit wöchentlich im Mittel $1000 \mathrm{mg} 5$-FU beträgt die mediane Überlebenszeit aller Stadien (Lausanne) 14 Monate, die 1-Jahresüberlebensrate $76 \pm 16 \%$ (act. surv.). Die Dosierung nach der individuellen Toleranz bei vorsichtiger Dosiseskalation ergab eine minimale Toxizität, erhöhte die Lebensqualität bei sicherer und schonender Palliation.

Schlüsselwörter: Disseminierte Lebermetastasen - Intraarterielle Chemotherapie - Intermittierende, intraluminäre Hypoxie.

\section{Indikation und Ergebnisse der Resektion von Lebermetastasen bei Abdominaltumoren}

\author{
B. Reismann und J. Neitzel \\ Chirurgische Abteilung Kreiskrankenhaus, D-4920 Lemgo
}

\section{Indications and Results of Hepatic Resection of Metastatic Abdominal Tumors}

\begin{abstract}
Summary. In a series of 368 malignant abdominal tumors in 81 patients $(=22 \%)$ liver metastases were found during the primary operation. In 15 patients a simultaneous hepatic resection was performed; in 3 hepatic resections were done after discovery of metastases by CT or sonography during a follow-up study. No patient died because of the hepatic resection. Of 11 patients with colorectal tumors, 6 are still alive after $9-72$ months. Two of four patients who underwent a gastrectomy and simultaneous hepatic resection are alive after 9 and 17 months, respectively. The results are better with the lobectomy than with simple excision or segmentectomy. Key words: Hepatic resection - Metastases - Abdominal tumors.

Zusammenfassung. Bei 368 Ersteingriffen wegen abdomineller Malignome fanden sich in 81 Fällen $(=22 \%)$ Lebermetastasen. In 15 Fällen konnte beim gleichen Eingriff eine Leberresektion durchgeführt werden. Bei drei Patienten wurden während der Tumornachsorge durch CT oder Sonographie entdeckte Lebermetastasen reseziert. Keiner dieser Patienten starb infolge der Leberresektion. 6 von 11 Patienten mit colorectalen Tumoren überleben bisher 9 bis 72 Monate. Zwei von 4 Patienten mit Gastrektomie und simultaner Leberresektion leben seit 9 bzw. 17 Monaten. Die Ergebnisse der Lobektomie sind besser als die der Excision oder der Segmentresektion.
\end{abstract}

Schliisselwörter: Leberresektion - Metastasen - Abdominaltumoren. 